\title{
Stable flies, Stomoxys calcitrans L. (Diptera: Muscidae), improve offspring fitness by avoiding oviposition substrates with competitors or parasites
}

\section{Steve B. S. Baleba ( $\sim$ bbaleba@icipe.org )}

International Centre of Insect Physiology and Ecology https://orcid.org/0000-0001-8629-6954

Baldwyn Torto

International Centre of Insect Physiology and Ecology

Daniel Masiga

International Centre of Insect Physiology and Ecology

Merid N. Getahun

International Centre of Insect Physiology and Ecology

Christopher W. Weldon

Department of Zoology and Entomology/Pretoria University

Research article

Keywords: Stomoxys calcitrans, oviposition, competitors, parasites, offspring fitness

Posted Date: May 20th, 2019

DOl: https://doi.org/10.21203/rs.2.9487/v1

License: (9) This work is licensed under a Creative Commons Attribution 4.0 International License. Read Full License

Version of Record: A version of this preprint was published at Frontiers in Ecology and Evolution on January 28th, 2020. See the published version at https://doi.org/10.3389/fevo.2020.00005. 


\section{Abstract}

Background Oviposition site selection by gravid female insects is an important determinant in species distribution, abundance, and population dynamics. Females may assess the suitability of a potential oviposition substrate by using cues from conspecific or heterospecific individuals already present. Here, we assessed whether the presence of conspecific, heterospecific larvae and parasites influenced oviposition decisions by the stable fly, Stomoxys calcitrans (Linneaus). Methods and Results Using dual and multiple-choice oviposition bioassays, we found that gravid female S. calcitrans avoided substrates with conspecific larvae, the larvae of house flies, Musca domestica (Linneaus), and the mite Macrocheles muscaedomesticae (Scopoli). Avoidance of conspecific and heterospecific larvae persisted in the dark, suggesting that this behaviour is mediated by olfactory cues. When we reared $\mathrm{S}$. calcitrans in the presence of conspecific larvae and the larvae of house flies at different densities we found that this negatively affected emergence time, larval weight, larval survival, pupal weight, pupal survival, and adult weight. We also demonstrated that individuals of S. calcitrans developed in the presence of mites exhibited low egg hatchability, and poor larval and adult survival. Conclusion Our study provides additional support for the "preference-performance" hypothesis in S. calcitrans, with gravid females preferring to lay eggs on a substrate that will enhance offspring fitness. We recommend that the olfactory cues involved in avoidance by gravid female $\mathrm{S}$. calcitrans of substrates with conspecific and heterospecific larvae should be elucidated. This could lead to the discovery of repellent chemicals important for S. calcitrans management.

\section{Background}

Oviposition site selection is a complex task in the life history of all holometabolous insects. As the egg stage is immobile and most adult insects do not practice maternal care, gravid females need to lay eggs on a substrate that maximises the fitness of their offspring. To select an appropriate breeding site, gravid females are guided by visual, mechanical and chemical cues [1]. In addition to these, intra- and interspecific competition [2] and parasitism [3,4] are also known to influence oviposition decisions by gravid female insects. This influence can be either facilitatory, neutral or inhibitory [5]. Facilitation of oviposition occurs when the presence of conspecific or heterospecific individuals in a specific substrate is an indicator of high quality habitat [6]. For example, Gonzalez et al. [7] demonstrated that female Aedes aegyptii and Aedes albopictus (Linneaus) preferred to oviposit into water containing conspecific larvae. A neutral effect is evident when there is no observable cost of sharing the same habitat with other individuals. This has been shown in Pieris napi (Linneaus), where females laid an approximately equal number of eggs when exposed to substrates with and without conspecific cues [8].

Inhibition of oviposition results when females are deterred from laying eggs on a substrate by the presence of competitors. Gravid females may avoid competition for their offspring through direct, aggressive interactions with other females, the marking and detection of larval resources with oviposition 
pheromones, or avoidance of cues associated with eggs or larvae already present. For example, females of the olive fruit fly, Bactrocera oleae (Rossi), display aggressive behaviour towards each other (wing waving, fast running towards the opponent, pouncing and boxing on the head and thorax of the foe) when they are close to oviposition sites [9]. In the apple maggot, Rhagoletis pomonella (Walsh), females avoid larval competition by marking their oviposition site with a pheromone that deters most females from laying eggs [10]. In the convergent lady beetle, Hippodamia convergens (Guérin-Méneville), gravid females lay more eggs on cleaned sorghum plants compared to those bearing conspecific and heterospecific larvae of the wheat aphid, Schizaphis graminum (Rondani) [2]. The inhibitory effect is mainly explained by the avoidance by insect gravid females of larval competition, which can reduce offspring growth, negatively affect survival [11], and sometimes increase predatory cannibalism [12,13] due to the over-exploitation of resources. Similarly, inhibition of insect oviposition behaviour can also be triggered by the presence of parasites. By laying more eggs on a substrate devoid of parasites, gravid female insects protect their offspring. This is evident in Drosophila melanogaster (Meigen), where females prefer to oviposit on alcohol-laden food sources that protect hatched larvae from wasp parasitisation [4].

Stable flies, Stomoxys calcitrans (Linneaus), are cosmopolitan blood-feeders that mechanically transmit viruses (e.g., West Nile fever virus, Rift Valley fever virus), bacteria (e,g., Bacillus anthracis, Pasteurella multocida), protozoans (e.g., Trypanosoma evansi, Besnoitia besnoit), and helminths (e.g., Habronema microstoma, Dirofilaria repens) to their hosts. Hosts include cattle, camels, horses, dogs, and humans [14]. During periods of high abundance, S. calcitrans can reduce weight gain in cattle by up to $19 \%$, and lead to a $40-60 \%$ reduction in milk yields $[15,16]$. In the USA, Taylor et al. [17] estimated that $S$. calcitrans lead to economic losses of around $\$ 2.2$ billion per year.

Gravid females of $S$. calcitrans use vertebrate herbivore dung for oviposition [18], as well as rotting plant material, such as silage, hay, grass clippings, and garden compost $[19,20]$. Decaying plant-based material is also an important ecological niche for other invertebrate species [21] such as the house fly, Musca domestica (Linneaus) [22] and the mite Macrocheles muscaedomesticae (Scopoli) [23]. Similar to $S$. calcitrans, gravid females of $M$. domestica seek manure, decaying vegetation and compost for egg deposition [24]. Macrocheles muscaedomesticae is a free-living cosmopolitan mite inhabiting decaying organic matter, including manure [25]. This mite feeds on the eggs and early larvae stages of flies [26]. These mites are also found attached to the abdomen of adult flies, which they infect and parasitise during adult fly emergence from the puparium. This interaction has long been considered phoretic, but it is suspected that mites can extract haemolymph from their hosts, thereby reducing their flight performance [27]. Macrocheles mites harm their hosts in different ways. Polak and Markow [28] showed that these mites influenced the copulatory success in the fruit fly Drosophila nigrospiracula (Patterson and Wheeler). They demonstrated that males infested with two mites copulated less frequently than 
uninfested individuals. But after removing mites, previously infested males copulated as many times as flies with no history of infestation. Also, under laboratory condition, Polak [29] showed that Macrocheles mites negatively affected the survivorship of $D$. nigrospiracula. The authors also demonstrated that this mite infestation lengthened the pre-oviposition period and reduced the number of eggs laid by gravid female D. nigrospiracula [29].

In this study, we determined how competition and parasitism affected oviposition decisions by gravid female $S$. calcitrans. We hypothesised they would avoid substrates with conspecific heterospecific larvae and parasites to protect their offspring from intra- or inter-specific competition and parasitism. To test this hypothesis, we first assessed whether the presence of $S$. calcitrans larvae, $M$. domestica larvae and M. muscaedomesticae adults in a breeding substrate could influence oviposition decisions by $S$. calcitrans. Next, we addressed the ultimate evolutionary question of the fitness costs incurred by $S$. calcitrans immature stages when developing on a substrate with high competition or parasitism. These results provide insights into how gravid females of $S$. calcitrans are likely to behave in the presence of conspecific and heterospecific larvae, or parasites on a substrate. Such information is important for the management of $S$. calcitrans because it could lead to the discovery of semiochemicals acting either as attractants or repellents.

\section{Methods}

\section{Study animals and substrates}

Stomoxys calcitrans flies were obtained from a single culture established for approximately one year at the Duduville campus of the International Centre of Insect Physiology and Ecology (icipe) in Nairobi, Kenya ( $1^{\circ} 13^{\prime} 12^{\prime \prime} S, 36^{\circ} 52^{\prime} 48^{\prime \prime} \mathrm{E} ; \approx 1600 \mathrm{~m}$ above sea level). Prior to the experiment, gravid females of $S$. calcitrans from the established colony were exposed to rabbit dung (fermented in a plastic bag for 1 week) placed in plastic containers $(21.5 \times 14.5 \times 7.4 \mathrm{~cm})$ for oviposition. Rabbit dung were collected from the icipe rabbit breeding unit. After exposure for $24 \mathrm{hrs}$ we transferred these containers to another cage $(75 \times 60 \times 45 \mathrm{~cm})$, and we monitored the development of the larval and pupal stages until adult emergence. Emerged flies were fed twice per day (0800 and 1600 hours) on defibrinated bovine blood obtained from a local abattoir (Farmers choice slaughterhouse, Kahawa west, Nairobi ( $\left.1^{\circ} 11^{\prime} 10.0^{\prime \prime} \mathrm{S}, 36^{\circ} 54^{\prime} 38.3^{\prime \prime} \mathrm{E}\right)$ ) poured on moistened cotton. Larvae of $M$. domestica were from a culture established from wild individuals captured from Kapiti Plain in Machakos County, Kenya ( $\left.1^{\circ} 37^{\prime} 60^{\prime \prime S}, 37^{\circ} 0^{\prime} 0^{\prime \prime E}\right)$, using Vavoua traps [30]. They were reared on rabbit dung as previously described, but adults were fed on rotten banana, orange and watermelon. Macrocheles muscaedomesticae (Fig. 5Ai) were harvested directly from unsterilized rabbit dung collected at the icipe rabbit breeding unit. We identified this mite following the morphological characteristics described by Özbek et al. [31] and Kamaruzaman et al. [32]. Insect rearing and experiments were done in a laboratory under buffered conditions of $25 \pm 5^{\circ} \mathrm{C}$ and $65 \pm 5 \%$ relative humidity. The photoperiod was 12L:12D unless otherwise stated. 


\section{Oviposition in the presence of conspecific and heterospecific larvae}

We performed multiple-choice oviposition bioassays in cages $(75 \times 60 \times 45 \mathrm{~cm})$. In each cage, we allowed 20 gravid female $S$. calcitrans to oviposit on four types of substrate presented simultaneously: (1) rabbit dung, (2) rabbit dung with ten S. calcitrans third instar larvae, (3) rabbit dung with ten M. domestica third instar larvae, and (4) rabbit dung with ten $S$. calcitrans and ten $M$. domestica third instar larvae. For each replicate, we counted the number of eggs laid on each treatment $24 \mathrm{hrs}$ after setting-up the bioassay. During all experiments performed in the laboratory, we used $50 \mathrm{~g}$ of rabbit dung, introduced in transparent $200 \mathrm{ml}$ plastic cups. The high walls of these cups prevented escape of larvae from the dung. These bioassays were replicated ten times.

\section{Oviposition in response to abundance of conspecific and heterospecific larvae}

In the previous bioassay, we found that gravid female $S$. calcitrans avoided laying eggs on a substrate containing conspecific larvae (Fig. 1A). Here, we aimed to establish whether avoidance of conspecifics was dependent on the number of individuals present on a substrate. We performed a density-dependent response oviposition bioassay where we exposed 20 gravid female $S$. calcitrans to five rabbit dung substrates (as described previously) containing an increasing number $(0,10,20,30$ and 40$)$ of conspecific third instar larvae. We found that female $S$. calcitrans avoided all the substrates with conspecific larvae (Fig. 1Bi). Afterwards, we assessed whether visual or chemical cues mediated conspecific avoidance. To do so, we followed the protocol developed by Yang and Shiao [33] in two blow flies, Chrysomya megacephala (Fabricus) and Chrysomya rufifacies (Macquart). This involved two-choice bioassays in darkness (OL: 24D photocycle) performed in a different climate room to the one where the culture was held so that the diurnal rhythm of the culture was not affected. In a cage $(34 \times 34 \times 34 \mathrm{~cm})$ containing ten gravid female $S$. calcitrans, we introduced two treatments: (1) rabbit dung and (2) rabbit dung+40 third instar $S$. calcitrans larvae. We found that gravid female $S$. calcitrans significantly avoided the substrate containing conspecific larvae (Fig. 1Bii). We also assessed whether gravid female $S$. calcitrans could avoid a substrate already used by conspecific larvae. In a two-choice bioassay, we allowed gravid female $S$. calcitrans to choose between used (where $40 \mathrm{~S}$. calcitrans eggs were reared until the pupae stage and removed) and unused substrates (one-week fermented rabbit dung). Each bioassay described above was performed for $24 \mathrm{hrs}$ after which we counted the number of eggs laid on each substrate treatment. Each bioassay was replicated ten times.

The same series of experiments was then performed using $M$. domestica larvae. In the multiple-choice oviposition bioassay, we found that gravid females of $S$. calcitrans laid the number of eggs laid on the control substrate and the substrate with larvae of $M$. domestica did not differ significantly (Fig. $1 \mathrm{~A}$ ). We 
therefore sought to elucidate whether this behaviour could change if the number of $M$. domestica larvae on the substrate increased, and to assess the role of visual or chemical cues in observed oviposition patterns using the same protocol as described for $S$. calcitrans.

\section{Oviposition in the presence of parasites}

To evaluate how oviposition by S. calcitrans is affected by the presence of parasites in dung, we performed two-choice bioassays. Ten gravid female $S$. calcitrans were introduced to a cage $(34 \times 34 \times 34$ $\mathrm{cm}$ ) containing a rabbit dung control and rabbit dung supplemented with 50 adults of the mite Macrocheles muscaedomesticae. We counted the number of eggs laid on each substrate after $24 \mathrm{hrs}$ and we replicated the experiment 10 times. We found that $S$. calcitrans gravid females laid fewer eggs on the substrate with mites (Fig. 2A). To find out if this behaviour was driven by visual or olfactory cues, we repeated the two-choice bioassays in darkness as described previously. Ten replicates of these bioassays were performed:

\section{Costs of developing on a substrate with conspecific and heterospecific larvae}

Danchin and Wagner [34] postulated that individuals developing in groups would pay a fitness cost due to increased competition for limited resources. Therefore, we hypothesised that $S$. calcitrans offspring developing with conspecific or heterospecific larvae at high density would exhibit poor development. To assess the effect of intraspecific competition on S. calcitrans, we reared first instar larvae in groups of 5 , 15 and 25 in plastic cups filled with $50 \mathrm{~g}$ of rabbit dung. Daily, we monitored the development of these larvae until adult emergence by recording the following parameters: (1) emergence time (from first instar larvae to adult emergence), (2) larval weight (weighed at 4, 7 and 10 days after the beginning of the experiment), (3) pupation percentage (equivalent to larval mortality), (4) pupal weight, (5) emergence percentage (equivalent to pupal mortality), and (6) adult weight. We replicated each treatment ten times. For the larvalw weight parameter, we individually weighed all the larvae present on each density treatment across times.

To test the fitness consequences for $S$. calcitrans developing in the presence of interspecific competition, we combined 10 first instar larvae of $S$. calcitrans with an incremental number of first instar $M$. domestica larvae as follows: 10:0, 10:10, 10:20, 10:30, 10:40. Each combination was introduced to $50 \mathrm{~g}$ of rabbit dung and replicated ten times. Here, we also monitored the development of $S$. calcitrans larvae until adult emergence by recording the same six fitness parameters listed previously. Owing to our inability to differentiate $S$. calcitrans larvae from $M$. domestica larvae on day 4 and 7 , we weighed $S$. calcitrans larvae only on day 10 . On day 10, S. calcitrans larvae were smaller, with translucent bodies, in comparison with $M$. domestica larvae, which were larger and pale white to yellow in colour. 


\section{Cost of developing in a substrate with parasites}

We hypothesised that if $S$. calcitrans eggs, larvae, or pupae were transferred to a substrate hosting the mite $M$. muscaedomesticae, egg hatchability, larval mortality and adult survival in $S$. calcitrans would be affected. We introduced ten eggs of $S$. calcitrans in transparent plastic cups of $200 \mathrm{ml}$ prior containing 50 $\mathrm{g}$ of rabbit dung infested with 50 adult mites of $M$. muscaedomesticae. As a control, we transferred ten eggs of $S$. calcitrans to rabbit dung without mites. Three days after, we determined the number of eggs that hatched by counting the number of larvae (L1 stage) found on each substrate treatment. To assess the effect of mite infestation on S. calcitrans larval survival, we introduced $10 \mathrm{~L} 1$ larvae of $S$. calcitrans to rabbit dung hosting mites as previously described. For the control treatment, we also reared $10 \mathrm{~L} 1$ larvae on substrates without mites. In both treatments, we counted the number of dead larvae and the number of pupae. To determine adult survival, we transferred $S$. calcitrans pupae on rabbit dung infested with mites to initiate parasitation during adult emergence. After emergence, we collected ten individuals of $S$. calcitrans loaded with at least five mites on the abdomen, and we transferred them to another cage $(20 \times 15 \times 14.5 \mathrm{~cm})$. As a control, we used ten new emerged healthy flies (without mites). For eight days, we supplied flies of each treatment (infested and non-infested) with defibrinated bovine blood daily and counted the number of dead individuals. We replicated the bioassay assessing egg hatchability and the larvae survival ten times, whereas we replicated the bioassay testing adult survival five times.

\section{Data analysis}

All analyses were performed using the R environment for statisitcal computing (version 3.5.1) [35]. The number of eggs laid in the presence of conspecific and heterospecific larvae, and with increasing abundance of $S$. calcitrans or $M$. domestica larvae, were not normally distributed (Shapiro-Wilk test: $p<0.05)$. For this reason, we used Kruskal-Wallis tests to compare oviposition among presented substrates, followed by Dunn's post hoc tests to identify homogenous subsets. Eggs laid under dark conditions, and with used or unused substrates were also not normally distributed. To compare oviposition under these conditions, we used the paired Mann-Wilcoxon test. Number of eggs laid by $S$. calcitrans in the presence or absence of the mite M. muscaedomesticae were normally distributed, whether tested in the light or dark. To analyse these data, we used paired t-tests.

In our experiment assessing the effects of intraspecific competition on fitness parameters of $S$. calcitrans, emergence time and pupal weight data were not normally distributed (Shapiro-Wilk test: $p<0.05)$. Due to this we used Kruskal-Wallis tests followed by Dunn's post hoc tests to see how emergence time and pupal weight varied across the tested larval densities. For the larval weight, except weight recorded at day 7 , which was not normally distributed (we used the Kruskal-Wallis test followed by Dunn's post hoc test for 
analysis), data from larval weight recorded on days 3 and 10 followed a normal distribution (Shapiro-Wilk test: $p>0.05$ ). Consequently, we ran analyses of variance (ANOVA) followed by Dunnett-Tukey-Kramer (DTK) post hoc multiple comparisons tests (due to the inequality of the samples size) using the R library 'DTK' [36]. We also used ANOVA followed by DTK post hoc tests to compare adult weight across the different larval densities owing to their normal distribution. Due to the binary nature of pupation (pupated vs non-pupated)and adult emergence (emerged vs non-emerged), we used generalised linear models (GLM) with binomial distribution to test whether these variable were affected by larval density [37]. We established the significance of the model using analysis of deviance (chi-squared) tests. Using the "emmeans" R package [38], we ran Tukey's multiple comparisons tests to identify homogenous subsets in pupation and adult emergence among the larval densities.

In the bioassay testing the effect of interspecific competition on S. calcitrans fitness parameters,

emergence time and pupal weight data were not normally distributed (Shapiro-Wilk test: $p<0.05$ ), so we ran Kruskal-Wallis tests followed by Dunn's post hoc tests to see which $M$. domestica larval densities differed from each other. Larval and pupal weight data were normally distributed (Shapiro-Wilk test: p>0.05), so we ran ANOVA tests followed by DTK post hoc tests. We used a generalised linear model (GLM) with binomial distribution and the analysis of deviance test (With Chi-square test) followed by the Tukey's multiple comparisons tests to determine how pupation and the percentage emergence varied across the different larval densities.

For the bioassay aiming to assess the effect of mite infestation on egg hatchability and larval survival of S. calcitrans data were binary (hatched vs unhatched). For this reason, we used a generalised linear model (GLM) with binomial distribution and an analysis of deviance (chi-squared) test. To compare survivorship of adult flies with mites and without mites, we performed Kaplan-Meier survival analysis with the Mantel-Cox log-rank chi-squared test using the R package "survival" [39].

\section{Results}

\section{Oviposition in the presence of conspecific and heterospecific larvae}

Female $S$. calcitrans deposited significantly more eggs on rabbit dung without conspecific larvae than on the substrates with conspecific larvae (Fig. $1 \mathrm{~A} ; \mathrm{H}=11.45 \mathrm{df}=3, P=0.009$ ). This avoidance behaviour was not noted when the substrate was already colonised by the larvae of $M$. domestica. The number of eggs laid by female $S$. calcitrans on substrates with different conspecific larval density did not differ but was significantly lower than on uncolonized rabbit dung ( $\mathrm{Fig} .1 \mathrm{Bi}, \mathrm{H}=14.13, \mathrm{df}=4, P=0.007$ ). Under dark conditions, females completely avoided the substrate with conspecific larvae (Fig. 1Bii: $U=0, P<0.001$ ). 
They also preferred the unused substrate over the substrate used by conspecific larvae (Fig. 1Biii: $U=55$, $P=0.002)$.

An incremental increase in the number of heterospecific larvae colonising rabbit dung led to fewer eggs laid by female $S$. calcitrans. The number of eggs laid by female $S$. calcitrans was significantly lower on substrates with 20-40 M. domestica larvae than on a control or dung with 10 heterospecific larvae (Fig 1 $\mathrm{Ci}: \mathrm{H}=13.61, \mathrm{df}=4, P=0.008$ ). Under dark conditions, female $S$. calcitrans laid significantly fewer eggs on a substrate with $40 \mathrm{M}$. domestica larvae in comparison with the control (Fig 1Cii: U=55, $P=0.002$ ).

Similarly, significantly fewer eggs were laid on the substrate used for $M$. domestica development than the control (Fig. 1Ciii: U=42, $P=0.019$ ).

\section{Oviposition in the presence of parasites}

Deposition of eggs by $S$. calcitrans on rabbit dung without or with mites differed. In the experiment performed under light, gravid female $S$. calcitrans laid significantly more eggs on the substrate devoid of mites than the substrate with mites (Fig. $2 A: t=-2.63, d f=9, P=0.018$ ). Under dark conditions, the number of eggs laid by females did not differ between the two treatments (Fig. 2B: $t=-0.14, d f=9, P=0.82$ ).

\section{Costs of developing on a substrate with conspecific and heterospecific larvae}

Effect of intraspecific competition on S. calcitrans fitness parameters

Larvae reared in a group of 5 and 15 reached the adult stage significantly faster than those reared in a group of 25 ( $\mathrm{Fig} .3 \mathrm{~A} ; \mathrm{H}=7.73, \mathrm{df}=2, P=0.02$ ). Also, larval density significantly influenced larval weight (Day 4: $\mathrm{F}_{2-63}=3.5, P=0.035$; Day 7: $\mathrm{H}=15.60, \mathrm{df}=2, P<0.001$; Day $10: \mathrm{F}_{2-50}=20.91, P<0.001$ ). Larvae reared in a group of 5 , followed by those reared in a group of 15 were heavier in comparison with those reared in a group of 25 (Fig. 3B). Despite differences in the body weight of larvae, the percentage of larvae reaching the pupal stage did not differ statistically (Fig. $3 \mathrm{C}$ : $\mathrm{GLM}, \chi^{2}=2.27, \mathrm{~d} . \mathrm{f}=2, P=0.32$ ). Pupal weight differed significantly across the larval density treatments (Fig. 3D, $\mathrm{H}=98.4, \mathrm{df}=2, P<0.0001$ ), with pupal weight declining significantly with each incremental increase in larval density. The percentage of adults emerging from pupae reared in a group of 5 was significantly higher than at the higher larval densities (Fig. 3E; GLM, $\chi^{2}=17.06$, d. $f=2, P<0.001$ ). Larval density also significantly affected adult weight $\left(\mathrm{F}_{2-75}=4.50, P<0.05\right)$. Adults from larvae reared in a group of 5 and 15 weighed more than those reared in a group of 25 (Fig. 3F). 
Density of $M$. domestica larvae significantly affected the emergence time $(H=13.88, d f=4, P=0.007)$, larval weight $\left(\mathrm{F}_{4-104}=4.45, P=0.002\right)$, pupation percentage $\left(\mathrm{GLM}, \chi^{2}=44.51\right.$, d.f $\left.=4, P<0.001\right)$, pupal weight $(\mathrm{H}=35.54, \mathrm{df}=4, P<0.001)$, emergence percentage $\left(\mathrm{GLM}, \chi^{2}=24.38\right.$, d. $\left.f=4, P<0.001\right)$ and adult weight $\left(\mathrm{F}_{4-57}=7.95, P<0.001\right)$ of the ten $S$. calcitrans individuals present in each substrate treatment. In comparison with $S$. calcitrans larvae reared in the presence of $0,10,20$ and $30 \mathrm{M}$. domestica larvae, $S$. calcitrans larvae reared in the presence of 40 house fly larvae took longer to reach the adult stage (Fig. 4A). When S. calcitrans were reared with 30 or $49 \mathrm{M}$. domestica larvae their larval weight was less than in other treatments (Fig. 4B). The percentage of S. calcitrans larvae that reached the pupal stage was higher on the substrate devoid of house fly larvae (Fig. 4C). Individuals from the substrate without larvae or with ten M. domestica larvae had greater pupal weight (Fig. 4D) and emergence percentage (Fig. 4E) than those from other treatments. Adults from the substrate without house fly larvae were heavier than those reared from other treatments (Fig 4F).

\section{Cost of developing in a substrate with parasites}

The percentage of eggs that hatched was lower when placed on substrates hosting mites in comparison with substrates without mites (Fig $5 B: G L M, \chi^{2}=59.55, d f=1, P<0.001$ ). Survival of larvae transferred to a substrate with mites was lower than those transferred to a substrate devoid of mites (Fig. 5C: GLM, $\chi^{2}=$ $171.03, \mathrm{df}=1, P<0.001)$. Mite infestation of the substrate negatively affected adult survival (Fig. 5D; logrank test, $\left.\chi^{2}=6.4, P=0.011\right)$.

\section{Discussion}

The results obtained in this work showed that oviposition decisions by gravid female $S$. calcitrans are influenced by the presence of conspecific, heterospecific larvae, and parasites on a substrate. Gravid female $S$. calcitrans laid significantly fewer eggs on substrates containing conspecific and heterospecific larvae. We demonstrated that ten conspecific larvae were enough to trigger this avoidance, whereas in the presence of $M$. domestica, avoidance began when there were more than ten larvae. This suggests that gravid female $S$. calcitrans consider not only the density but identity of competitors when selecting oviposition sites. Similarly, Wachira et al. [40] demonstrated that substrate augmentation of Culex quinquefasciatus (Say) larval density significantly reduced the oviposition activity index of gravid female Anopheles gambiae. 
In darkness, S. calcitrans laid significantly fewer eggs in substrates with conspecific and M. domestica larvae, suggesting that instead of visual cues this avoidance is guided by olfactory cues. Further, avoidance of oviposition substrates continued after larvae had been removed from them. The oviposition-deterrent effect of conspecific- and heterospecific-larvae found in this work has been shown in several species of Diptera. In Culiseta longiareolata (Macquart), $88 \%$ of gravid females laid eggs in pools with low conspecific larval density in comparison with pools with a high larval density [41]. In Culex restuans (Theobald), Reiskind and Wilson [42] observed that gravid females avoided laying in containers with conspecific larvae. In $C$. megacephala, when liver with and without larvae of $C$. rufifacies were provided to gravid females, they oviposited on liver without larvae [33]. We suggest that $S$. calcitrans and M. domestica larvae may produce one or several chemical cues that strongly deter the oviposition behaviour of $S$. calcitrans gravid females. Several chemical cues from conspecific- and heterospecificlarvae acting as repellents have already been identified in various studies. For instance, the alkane nheneicosane found in the cuticle of $A$. aegypti larvae [43], deter oviposition of this species at 100 and $1000 \mathrm{mg} / \mathrm{l}$ [44]. Gravid female Aedes albopictus are also repelled by n-heneicosane (secreted by Ae. aegyptis larvae) at 30, 50, 100 and 200 ppm [43]. Also, several esters extracted from A. aegypti eggs (methyl dodecanoate, methyl tetradecanoate, methyl (Z)-9-hexadecenoate, methyl hexadecanoate (Z)-9hexadecenoic acid, methyl (Z)-9-octadecenoate, and methyl octadecanoate (Z)-9-octadecenoic acid) were found to repel $A e$. aegypti gravid females [44]. Frass produced by developing larvae may also represent a source of olfactory cues used by $S$. calcitrans to avoid substrates. Frass of conspecific- and heterospecific larvae has been found to repel a range of insects, including Cydalima perspectalis (Walker) [47], Monochamus alternatus (Hope) [48], Delia radicum (Linneaus) [49] and Ostrinia furnacalis (Guenée), Ostrinia scapulalis (Walker), and Ostrinia latipennis (Warren) [50]. Our observations offer the potential for discovery of repellent compounds that may be used to manage $S$. calcitrans and reduce the spread of diseases that they transmit.

It is important to note one caveat in interpreting our results that oviposition by $S$. calcitrans was reduced in dung where conspecific and heterospecific larvae had completed their development. In our study, we reared L1 larvae of $S$. calcitrans and $M$. domestica until the pupae stage and we used the rearing substrate for the bioassay. While we suggest a role for chemical cues persisting from larvae or their frass, it may be possible that lower oviposition on a used substrate is also affected by physicochemical properties of these substrates. In comparison with unused substrates, those previously occupied by $S$. calcitrans or $M$. domestica larvae may be less nutritious, have lower water content, and consequently be unsuitable for oviposition. We have shown previously that gravid female $S$. calcitrans oviposit on donkey and sheep dung, which have high nitrogen, phosphorous, potassium and zinc concentrations, in preference over camel and cow dung, which have lower concentrations of the same nutrients [18]. In the same study, optimal developmental time was associated with moderate dung water content, which was characteristic of the preferred dung types. In Junonia coenia (Hübner) gravid females prefer host-plants with high nitrogen levels [51]. To overcome this potential limitation, further studies are required with an additional treatment of weathered dung in which no larvae have developed.

Page $11 / 22$ 
We found that intra- or inter-specific competition negatively affected the fitness of $S$. calcitrans offspring. This provides a functional basis for avoidance of substrates with conspecific or heterospecific larvae by gravid female $S$. calcitrans. The impact of larval density on fitness parameters has also been found in other Diptera including Hermetia illucens [52], B. oleae [53], D. melanogaster [54], Ae. albopictus [11] and Ae. aegypti [55]. When resources are limited, larval crowding induces exploitative competition that lengthens larval developmental time, increases larval mortality, and reduces larval, pupal and adult weight $[49,56,57]$. This effect is mainly attributed to a reduction in the share of nutrients available in the breeding substrate for individuals. For illustration, Dutra et al [58] observed that glycogen concentration in Ae. aegypti larvae decreased by almost $50 \%$ as larval density increased. In $H$. illucens, crude fat concentration is higher in larvae reared at low density than those reared at high density [52]. Other studies suggest that high larval mortality observed with increased larval density results from interference competition. In crowded situations, $D$. melanogaster larvae exhibit cannibalism by killing and consuming other larvae [12,13].

Our study revealed that gravid female $S$. calcitrans were able to distinguish between substrates hosting parasites from substrates without them. In the light, gravid female $S$. calcitrans avoided the substrate containing the mite M. muscaedomesticae. Avoidance of parasitized substrates for oviposition is also found in other insect groups. Sadek et al. [3] demonstrated that the moth Spodoptera littoralis (Biosduval) preferred to oviposit on alfafa compared to cotton where their eggs are more likely to be parasitized by the wasp, Chelonus inanitus (Linneaus). In darkness, we observed that gravid female S. calcitrans deposited the same number of eggs on substrates with and without mites. This indicates that rather than olfactory cues, $S$. calcitrans may use visual cues to detect mites on the substrate. In D. melanogaster, Kacsoh et al [4] showed that oviposition in an alcohol-laden food source that protects hatched larvae from wasps was mediated by visual cues. As in the case of substrate colonisation by con-and heterospecific larvae, avoidance of M. muscaedomesticae also benefited the fitness of S. calcitrans offspring. We found that egg hatchability, and larval and adult survival were higher in substrates without the mite M. muscaedomesticae. Axtell $[26,57]$ reported that this mite could kill three to four $S$. calcitrans eggs per day. As well as hemolymph consumption when attached to larvae or adults, it is also suspected that on the host, the mite also induces melanisation which is an immune response involving the enzyme phenoloxidase. González-Santoyo and Córdoba-Aguilar [59] indicated that the production of this enzyme has a fitness cost in the host. We suggest that these two factors contributed to higher mortality of $S$. calcitrans larvae and adults in the presence of mites. As such, M. muscaedomesticae may represent a viable biological control agent for $S$. calcitrans.

In summary, our results indicate that gravid female $S$. calcitrans avoid substrates colonised by conspecific larvae, M. domestica larvae and the mite M. muscaedomesticae. This is likely due to the high 
fitness costs of competition and parasitisation for their offspring. We believe that the avoidance of substrates with conspecific and heterospecific larvae in $S$. calcitrans is mediated by olfactory cues. Therefore, we recommend that additional studies be conducted to identify the chemical cues implicated in this avoidance behaviour and their source. This will aid in development of improved control strategies against $S$. calcitrans.

\section{Declarations}

\section{Ethics approval and consent to participate}

Not applicable

\section{Consent for publication}

Not applicable

\section{Availability of data and material}

All data collected during this study will be archived in the Dryad data repository upon acceptance

\section{Competing interests}

The authors whose names are listed immediately below certify that they have no affiliations with or involvement in any organization or entity with any financial interest, or non-financial interest in the subject matter or materials discussed in this manuscript.

\section{Funding}

We thank Deutscher Akademischer Austauschdienst (DAAD) that provided Steve B. S. Baleba with a doctoral scholarship through the ARPPIS-DAAD scholarship programme. This work was supported by the IBCARP camel, grant no. DCI-FOOD/2014/ 346-739 - mainly by European Union. We also gratefully acknowledge the financial support for this research by the following organizations and agencies: Swedish International Development Cooperation Agency (Sida); UK Department for International Development (DFID); the Swiss Agency for Development and Cooperation (SDC); and the Kenyan Government. The views expressed herein do not necessarily reflect the official opinion of the donors. The funders had no role in study design, data collection and interpretation, or the decision to submit the work for publication. 


\section{Authors contribution}

SBSB: conceived the research idea, designed the experiment, collected and analysed the data, wrote the manuscript; DM: supervised and proofread the manuscript; BT: supervised and proofread the manuscript; MN. Getahun: designed the experiment, supervised and assisted with manuscrpt preparation; CWW: designed the experiment, supervised and assisted with manuscrpt preparation. All authors have read and approved the manuscript.

\section{References}

1. Bentley MD, Day JF. Chemical ecology and behavioral aspects of mosquito oviposition. Annual review of entomology. 1989; 34:401-421.

2. Michaud JP, Jyoti JL. Repellency of conspecific and heterospecific larval residues to Hippodamia convergens (Coleoptera: Coccinellidae) ovipositing on sorghum plants. European Journal of Entomology. 2007; 104:399-405.

3. Sadek MM, Hansson BS, Anderson P. Does risk of egg parasitism affect choice of oviposition sites by a moth? A field and laboratory study. Basic and Applied Ecology. 2010; 11:135-43.

4. Kacsoh BZ, Lynch ZR, Mortimer NT, Schlenke TA. Fruit flies medicate offspring after seeing parasites. Science. 2013; 339:947-50.

5. Prokopy RJ, Roitberg BD. Prokopy RJ, Roitberg BD. Joining and avoidance behavior in nonsocial insects. Annual Review of Entomology. 2001; 46:631-65.

6. Köhncke A. When and where to lay your eggs? Humboldt-Universität zu Berlin, MathematischNaturwissenschaftliche Fakultät l; 2013.

7. Gonzalez PV, González Audino PA, Masuh HM. Oviposition behavior in Aedes aegypti and Aedes albopictus (Diptera: Culicidae) in response to the presence of heterospecific and conspecific larvae. Journal of Medical Entomology. 2016; 53:268-72.

8. Raitanen J, Forsman JT, Kivelä SM, Mäenpää MI, Välimäki P. Attraction to conspecific eggs may guide oviposition site selection in a solitary insect. Behavioral Ecology. 2014; 25:110-6.

9. Benelli G. Aggressive behavior and territoriality in the olive fruit fly, Bactrocera oleae (Rossi) (Diptera: Tephritidae): Role of residence and time of day. Journal of Insect Behavior. 2014; 27:145-61.

10. Averill AL, Prokopy RJ. Intraspecific competition in the Tephritid fruit fly Rhagoletis Pomonella. Ecology. 1987; 68:878-86.

11. Yoshioka M, Couret J, Kim F, McMillan J, Burkot TR, Dotson EM, et al. Diet and density dependent competition affect larval performance and oviposition site selection in the mosquito species Aedes albopictus (Diptera: Culicidae). Parasites \& Vectors. 2012; 5:225.

12. Vijendravarma RK, Narasimha S, Kawecki TJ. Predatory cannibalism in Drosophila melanogaster larvae. Nature Communications. 2013; 4:2744 
13. Ahmad M, Chaudhary SU, Afzal AJ, Tariq M. Starvation-induced dietary behaviour in Drosophila melanogaster larvae and adults. Scientific Reports. 2015; 5:14285.

14. Baldacchino F, Muenworn V, Desquesnes M, Desoli F, Charoenviriyaphap T, Duvallet G. Transmission of pathogens by Stomoxys flies (Diptera, Muscidae): a review. Parasite. 2013; 20:26.

15. Carn VM. The role of dipterous insects in the mechanical transmission of animal viruses. British Veterinary Journal, 1996; 152:377-393.

16. Walker AR. Disease caused by arthropods. In Sewell MMH, Brocklesby DW (Eds.). Handbook on animal diseases in the tropics (4th edition), Bailliere Tindall: London. 1990.44.

17. Taylor DB, Moon RD, Mark DR. Economic impact of stable flies (Diptera: Muscidae) on dairy and beef cattle production. Journal of Medical Entomology. 2012; 49:198-209.

18. Baleba SBS, Torto B, Masiga D, Weldon CW, Getahun MN. Egg-laying decisions based on olfactory cues enhance offspring fitness in Stomoxys calcitrans L. (Diptera: Muscidae). Scientific Reports. 2019; 9:3850

19. Zumpt F. The Stomoxyine biting flies of the world: Diptera, Muscidae; taxonomy, biology, economic importance and control measures. Gustav Fischer Verlag, Stuttgart, Germany. 1973; 175.

20. Axtell RC. Fly control in confined livestock and poultry production. technical monograph. Ciba-Geigy Corporation Greensboro, NC. 1986; 1-59

21. Sladecek FXJ, Segar ST, Lee C, Wall R, Konvicka M. Temporal segregation between dung-inhabiting beetle and fly species. Lutermann H, editor. PLOS ONE. 2017;12: e0170426.

22. Broce $A B$, Haas MS. Relation of cattle manure age to colonization by stable fly and house fly (Diptera: Muscidae). Journal of the Kansas Entomological Society. 1999;60-72.

23. Jalil M, Rodriguez JG. Studies of behaviour of Macrocheles muscaedomesticae (Acarina: Macrochelidae) with emphasis on its attraction to the house fly. Annals of the Entomological Society of America. 1970; 63: 738-744.

24. Machtinger ET, Geden CJ, Hogsette JA, Leppla NC. Development and oviposition preference of house flies and stable flies (Diptera: Muscidae) in six substrates from Florida equine facilities. Journal of Medical Entomology. 2014; 51:1144-50.

25. Gerson U, Smiley RL, Ochoa R, Gerson U. Mites (acari) for pest control. 2nd ed. Oxford; Malden, MA: Blackwell Science; 2003.

26. Axtell RC. Acarina occurring in domestic animal manure1. Annals of the Entomological Society of America. 1963; 56:628-33.

27. Luong LT, Penoni LR, Horn CJ, Polak M. Physical and physiological costs of ectoparasitic mites on host flight endurance. Ecological Entomology. 2015; 40:518-24.

28. Polak M, Markow TA. Effect of ectoparasitic mites on sexual selection in a Sonoran Desert fruit fly. Evolution. 1995; 49:660-9.

29. Polak M. Polak M. Ectoparasitic effects on host survival and reproduction: The DrosophilaMacrocheles association. Ecology. 1996;77: 1379-89. 
30. Mihok S, Kang'ethe EK, Kamau GK. Trials of traps and attractants for Stomoxys spp. (Diptera: Muscidae). Journal of Medical Entomology. 1995; 32:283-9.

31. Özbek HH, Bal DA, Doğan S. The genus Macrocheles Latreille (Acari: Mesostigmata: Macrochelidae) from Kelkit Valley (Turkey), with three newly recorded mite species. Turkish Journal of Zoology. 2015; 39:768-80.

32. Kamaruzaman NAC, Mašán P, Velásquez Y, González-Medina A, Lindström A, Braig HR, et al. Macrocheles species (Acari: Macrochelidae) associated with human corpses in Europe. Experimental and Applied Acarology. 2018; 76:453-71.

33. Yang S-T, Shiao S-F. Oviposition preferences of two forensically important blow fly species, Chrysomya megacephala and C. rufifacies (Diptera: Calliphoridae), and implications for postmortem interval estimation. Journal of Medical Entomology. 2012; 49:424-35.

34. Danchin E, Wagner RH. The evolution of coloniality: the emergence of new perspectives. Trends in Ecology \& Evolution. 1997; 12:342-7.

35. R Core Team. R: A language and environment for statistical computing R Foundation for Statistical Computing, Vienna, Austria. URL https://www.R-project.org/.2018.

36. Matthew K, Lau. DTK: Dunnett-Tukey-Kramer pairwise multiple comparison test adjusted for unequal variances and unequal sample sizes. R package version 3.5. https://CRAN.Rproject.org/package=DTK. 2013

37. Warton DI, Hui FK. The arcsine is asinine: the analysis of proportions in ecology. Ecology. 2011; 92:3-10.

38. Russell Lenth. emmeans: Estimated Marginal Means, aka Least-Squares Means. R package version 1.2.2. https://CRAN.R-project.org/package=emmeans. 2018.39. Therneau T. _A package for survival analysis in S_. version 2.38, <URL: https://CRAN.R-project.org/package=survival>. 2015.

40. Wachira SW, Ndung'u M, Njagi PGN, Hassanali A. Comparative responses of ovipositing Anopheles gambiae and Culex quinquefasciatus females to the presence of Culex egg rafts and larvae. Medical and Veterinary Entomology. 2010; 24:369-74.

41. Kiflawi M, Blaustein L, Mangel M. Oviposition habitat selection by the mosquito Culiseta longiareolata in response to risk of predation and conspecific larval density. Ecological Entomology. 2003; 28:168-173.

42. Reiskind MH, Wilson ML. Culex restuans (Diptera: Culicidae) Oviposition behavior determined by larval habitat quality and quantity in south-eastern Michigan. Journal of Medical Entomology. 2004; 41:179-86.

43. Mendki MJ, Ganesan K, Prakash S, Suryanarayana MVS, Malhotra RC, Rao KM, Vaidyanathaswamy R. Heneicosane: an oviposition-attractant pheromone of larval origin in Aedes aegyptimosquito. Current Science. 2000; 78: 12951296.

44. Seenivasagan T, Sharma KR, Sekhar K, Ganesan K, Prakash S, Vijayaraghavan R. Electroantennogram, flight orientation, and oviposition responses of Aedes aegypti to the oviposition 
pheromone n-heneicosane. Parasitology Research. 2009; 104:827-33.

45. Gonzalez PV, Audino PAG, Masuh HM. Electrophysiological and behavioural response of Aedes albopictus to n-heinecosane, an ovipositional pheromone of Aedes aegypti. Entomologia Experimentalis et Applicata. 2014; 151:191-7.

46. Ganesan K, Mendki MJ, Suryanarayana MVS, Prakash S, Malhotra RC. Studies of Aedes aegypti (Diptera: Culicidae) ovipositional responses to newly identified semiochemicals from conspecific eggs. Australian Journal of Entomology. 2006; 45:75-80.

47. Molnár BP, Tóth Z, Kárpáti Z. Synthetic blend of larval frass volatiles repel oviposition in the invasive box tree moth, Cydalima perspectalis. Journal of Pest Science. 2017; 90:873-85.

48. Li S-Q, Zhang Z-N. Influence of larval frass extracts on the oviposition behaviour of Monochamus alternatus (Col., Cerambycidae). Journal of Applied Entomology. 2006; 130:177-82.

49. Jones $T$, and Finch S. The effect of a chemical deterrent, released from the frass of caterpillars of the garden pebble moth, on cabbage root fly oviposition. Entomologia Experimentalis et Applicata, 1987; 45: 283-288.

50. Li G, Ishikawa Y. Oviposition deterrents in larval frass of four Ostrinia species fed on an artificial diet. Journal of Chemical Ecology. 2004; 30:1445-56.

51. Prudic KL, Oliver JC, Bowers MD. Soil nutrient effects on oviposition preference, larval performance, and chemical defense of a specialist insect herbivore. Oecologia. 2005; 143:578-87.

52. Barragan-Fonseca KB, Dicke M, van Loon JJA. Influence of larval density and dietary nutrient concentration on performance, body protein, and fat contents of black soldier fly larvae (Hermetia illucens). Entomologia Experimentalis et Applicata. 2018; 166:761-70.

53. Burrack HJ, Fornell AM, Connell JH, O'Connell NV, Phillips PA, Vossen PM, et al. Intraspecific larval competition in the olive fruit fly (Diptera: Tephritidae). Environmental Entomology. 2009; 38:140010.

54. Durisko Z, Dukas R. Attraction to and learning from social cues in fruitfly larvae. Proceedings of the Royal Society B: Biological Sciences. 2013; 280:20131398-20131398.

55. Reiskind MH, Lounibos LP. Effects of intraspecific larval competition on adult longevity in the mosquitoes Aedes aegypti and Aedes albopictus. Medical and Veterinary Entomology. 2009; 23:628.

56. Sugiura S, Yamazaki K, Yamaura Y. Intraspecific competition as a selective pressure on the choice of oviposition site in a phytophagous insect: Intraspecific competition of a leaf miner. Biological Journal of the Linnean Society. 2007; 92:641-50.

57. Legros M, Lloyd AL, Huang Y, Gould F. Density-dependent intraspecific competition in the larval stage of Aedes aegypti (Diptera: Culicidae): Revisiting the current paradigm. Journal of Medical Entomology. 2009; 46:409-19.

58. Dutra HLC, Lopes da Silva V, da Rocha Fernandes M, Logullo C, Maciel-de-Freitas R, Moreira LA. The influence of larval competition on Brazilian Wolbachia-infected Aedes aegypti mosquitoes. Parasites \& Vectors. 2016 9:282 
59. González-Santoyo I, Córdoba-Aguilar A. Phenoloxidase: a key component of the insect immune system. Entomologia Experimentalis et Applicata. 2012; 142:1-16.

\section{Figures}
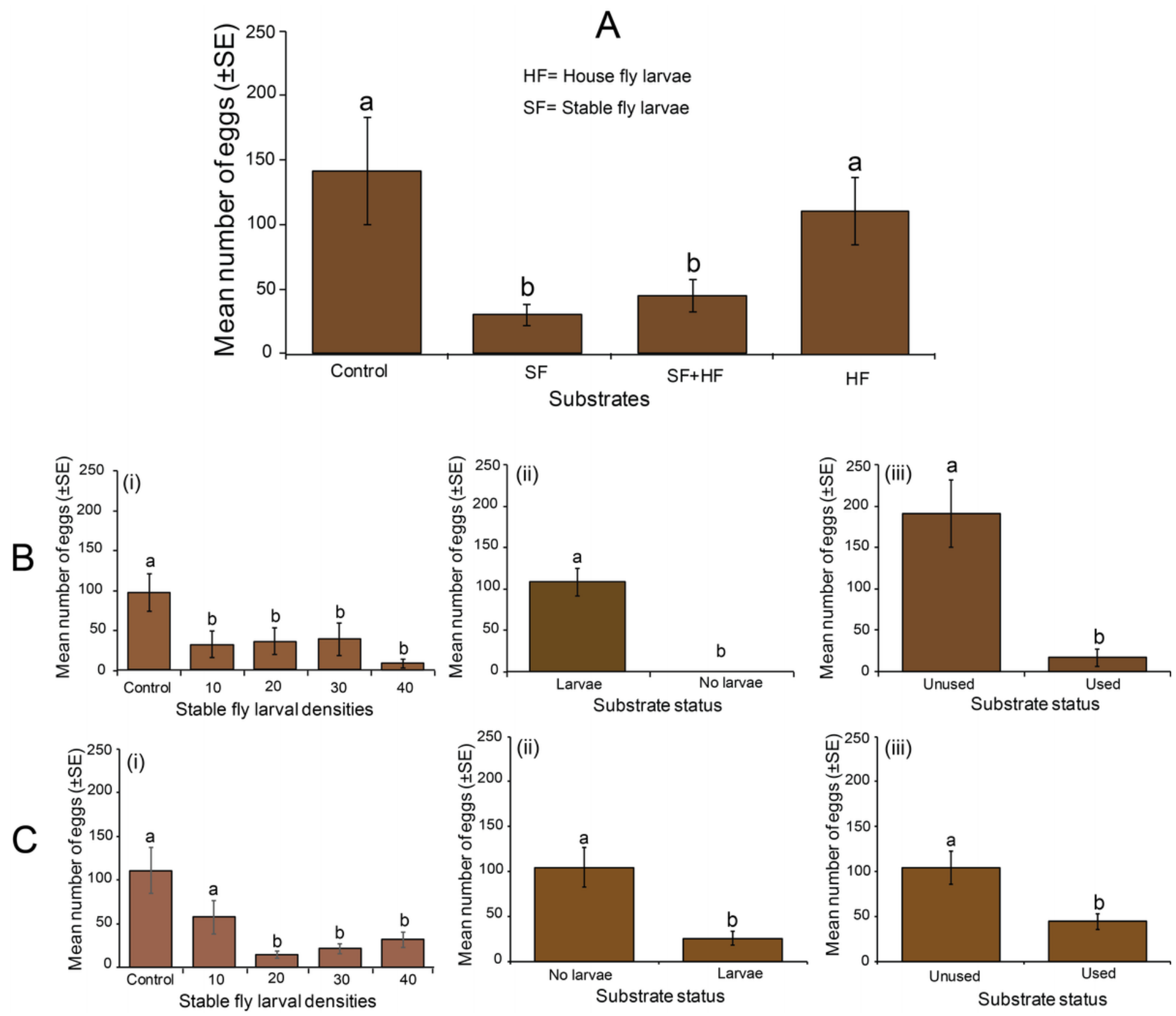

\section{Figure 1}

Influence of the presence of S. calcitrans and M. domestica larvae on oviposition decisions by S. calcitrans. (A) Bar chart showing the mean number of eggs laid by gravid female S. calcitrans in the presence of conspecific larvae and the larvae of the house fly (HF), M. domestica. (B) Bar charts illustrating the mean number of eggs laid by gravid female $\mathrm{S}$. calcitrans in the presence of conspecific larvae following three experimental conditions: (i) different larval densities, (ii) in darkness, (ii) with 
unused and used substrates. (C) Bar charts depicting the mean number of eggs laid by gravid female S. calcitrans in the presence of $\mathrm{M}$. domestica larvae following three experimental conditions: (i) different larval densities, (ii) in darkness, and (iii) with unused and used substrates. Error bars indicate the standard error of the mean. Treatments with different lowercase letters are significantly different from each other (Kruskal-Wallis test followed by Dunn's post hoc tests or Wilcoxon-Mann-Whitney tests, $\mathrm{P}<0.05, \mathrm{n}=10)$.
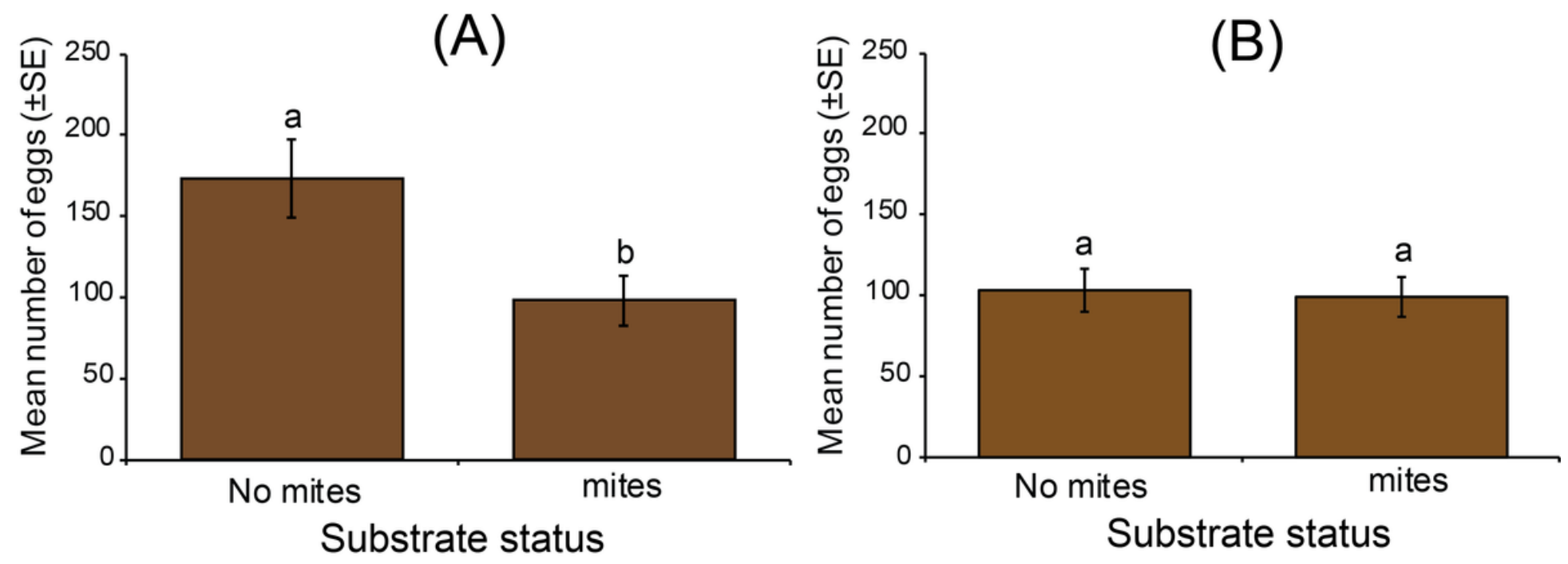

Figure 2

Influence of the presence of the mite Macrocheles muscaedomesticae on oviposition decisions by S. calcitrans. (A) Bar chart depicting the mean number of eggs laid by S. calcitrans on substrates with and without mites under light (Paired t-test, $P<0.05, n=10$ ). (A) Bar chart depicting the mean number of eggs laid by $S$. calcitrans on substrates with and without mites in darkness (Paired t-test, $P>0.05, n=10$ ). Error bars indicate the standard error of the mean. Treatments with different lowercase letters are significantly different from each other. 


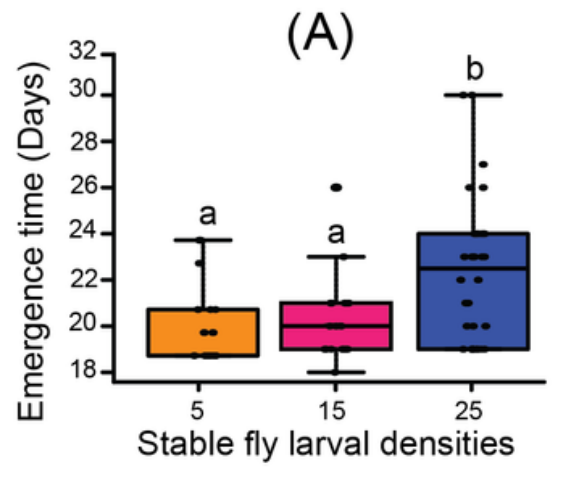

(D)

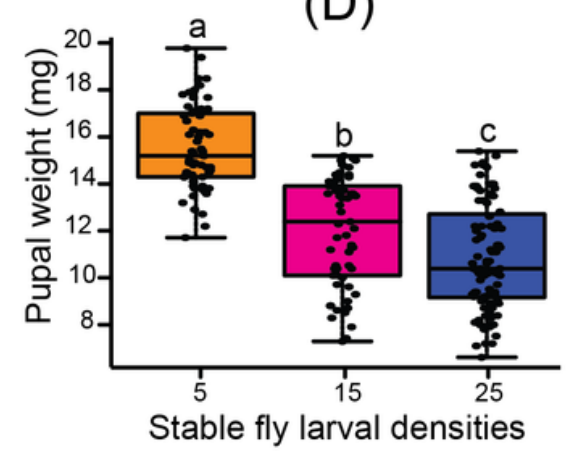

(B)

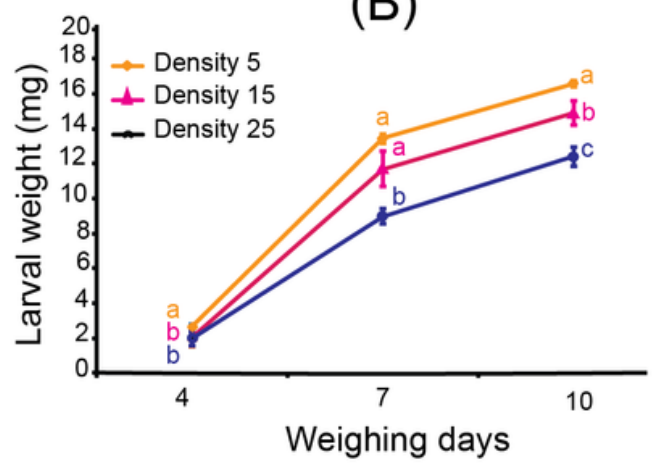

$(\mathrm{E})$

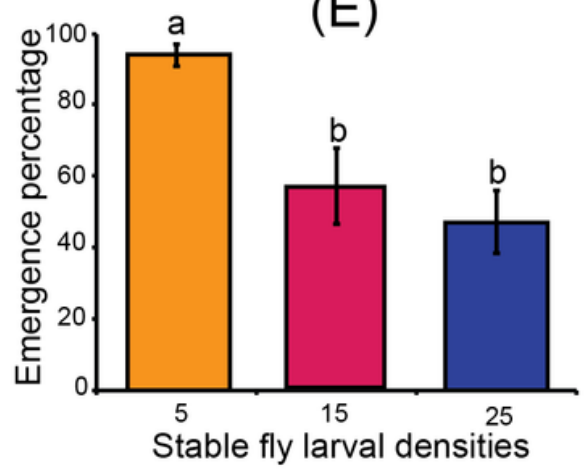

(C)

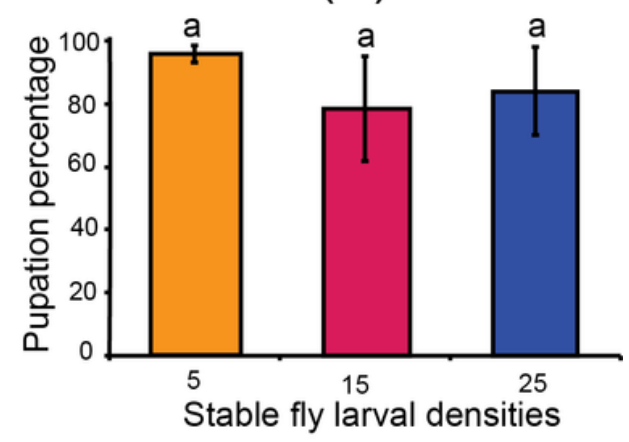

$(\mathrm{F})$

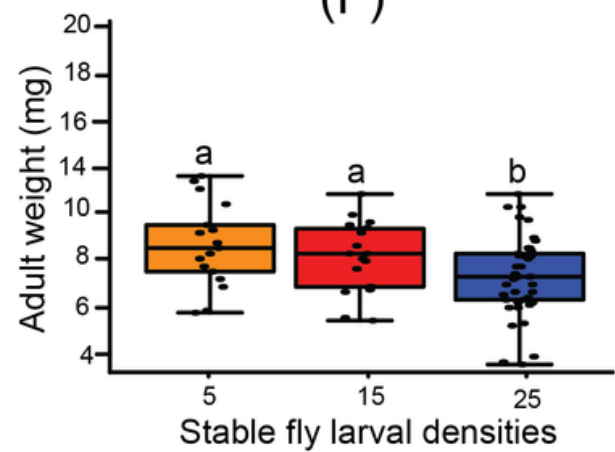

\section{Figure 3}

Effect of conspecific larvae density on fitness traits of S. calcitrans. (A) Boxplot showing S. calcitrans emergence time across the different larval densities (Kruskal-Wallis test followed by Dunn's post-hoc tests, $P<0.05, n=10$ ). (B) Line graph illustrating the change of $S$. calcitrans larvae weight across the different larval densities (ANOVA test followed by SNK's post hoc test, $P<0.05, n=10$ ), 7 (Kruskal-Wallis test followed by Dunn's post hoc test, $P<0.05, n=10$ ) and 10 (ANOVA test followed by SNK's post hoc test, $P<0.05, n=10$ ). (C) Bar charts depicting the mean percentage of $S$. calcitrans larvae reaching the pupal stage across the different larval densities (GLM with binomial distribution followed by Tukey post-hoc mean separation test, $P<0.05, n=10$ ). (D) Boxplot showing S. calcitrans pupal weight across the different larval densities (Kruskal-Wallis test followed by Dunn's post hoc test, $P<0.05, n=10$ ). (E) Bar chart illustrating the mean percentage of $\mathrm{S}$. calcitrans emerging as adults from the pupal stage across the different larval densities (GLM with binomial distribution followed by Tukey post hoc mean separation test, $P<0.05, n=10)$. (F) Boxplot depicting $S$. calcitrans adult weight across the different larval densities (ANOVA test followed by DTK's post hoc test, $P<0.05, n=10$ ). On each boxplot, the minimum and maximum values of all the data are represented by the ends of boxplot whiskers. On the line graph and bar charts, error bars indicate the standard error of the mean. Treatments with different lowercase letters are significantly different from each other. 


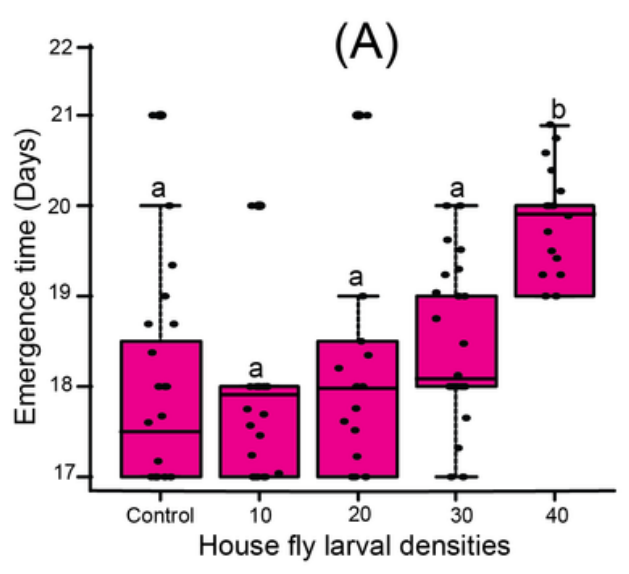

(D)

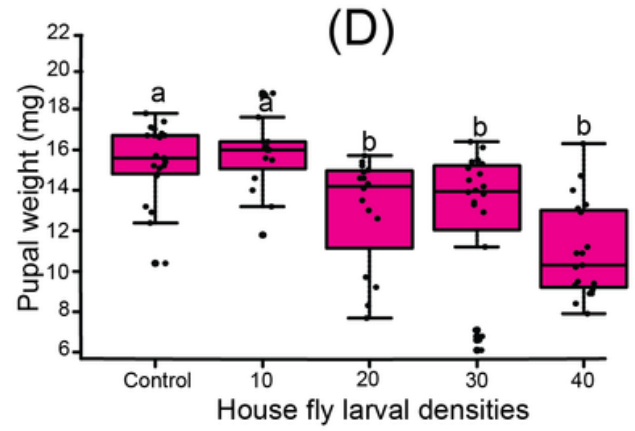

(B)

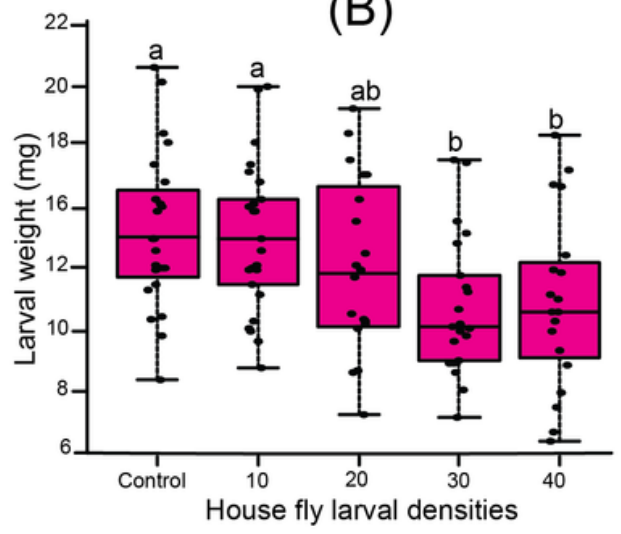

$(\mathrm{E})$

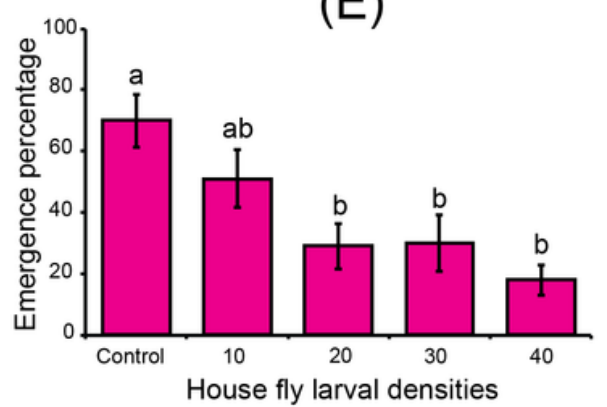

(C)

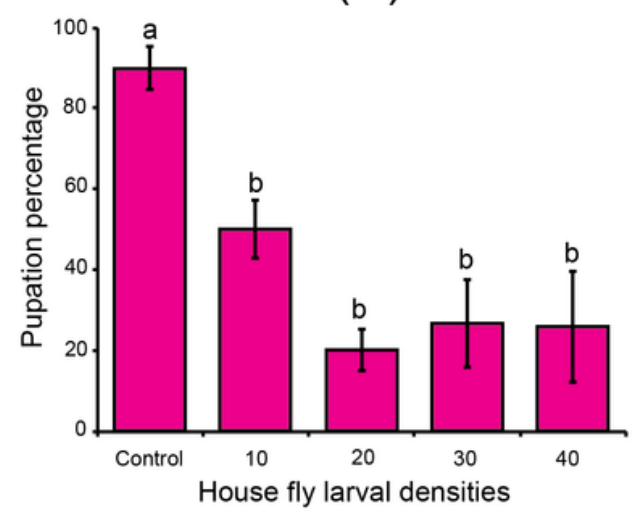

$(\mathrm{F})$

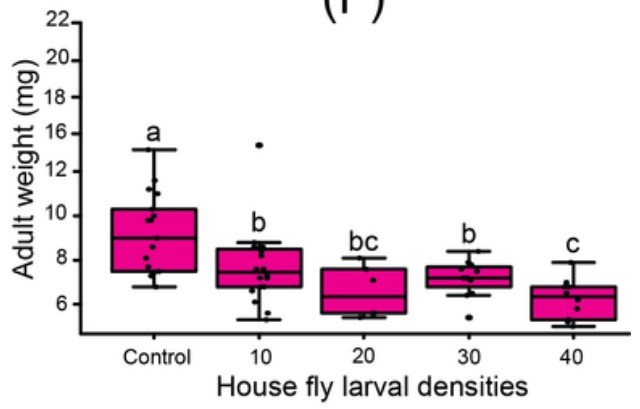

Figure 4

Effect of M. domestica larval density on fitness parameters of S. calcitrans. (A) Boxplot showing the variation of $\mathrm{S}$. calcitrans emergence time across the different larval densities (Kruskal-Wallis test followed by Dunn's post hoc test, $P<0.05, n=10$ ). (B) Boxplot illustrating the change of $S$. calcitrans larvae weight across the different larval densities (ANOVA test followed by DTK's post hoc test, $P<0.05, n=10$ ). (C) Histograms depicting the pupation percentages of S. calcitrans across the different larval densities (GLM with binomial distribution followed by Tukey post hoc mean separation test, $P<0.05, n=10$ ), (D) Bar chart showing the variation of $\mathrm{S}$. calcitrans pupae weight across the different larval densities (KruskalWallis test followed by Dunn's post hoc test, $P<0.05, n=10$ ). (E) Bar chart illustrating the variation of $S$. calcitrans emergence percentage across the different larval densities (GLM with binomial distribution followed by Tukey post hoc mean separation test, $P<0.05, n=10)$. (F) Boxplot depicting the variation of $S$. calcitrans adult weight across the different larval densities (ANOVA test followed by DTK's post hoc test, $P<0.05, n=10$ ). On each boxplot, the minimum and maximum values of all the data are represented by the ends of boxplot whiskers. On the bar charts, the error bars indicate the standard error of the mean (SEM). Treatments with different lowercase letters are significantly different from each other 

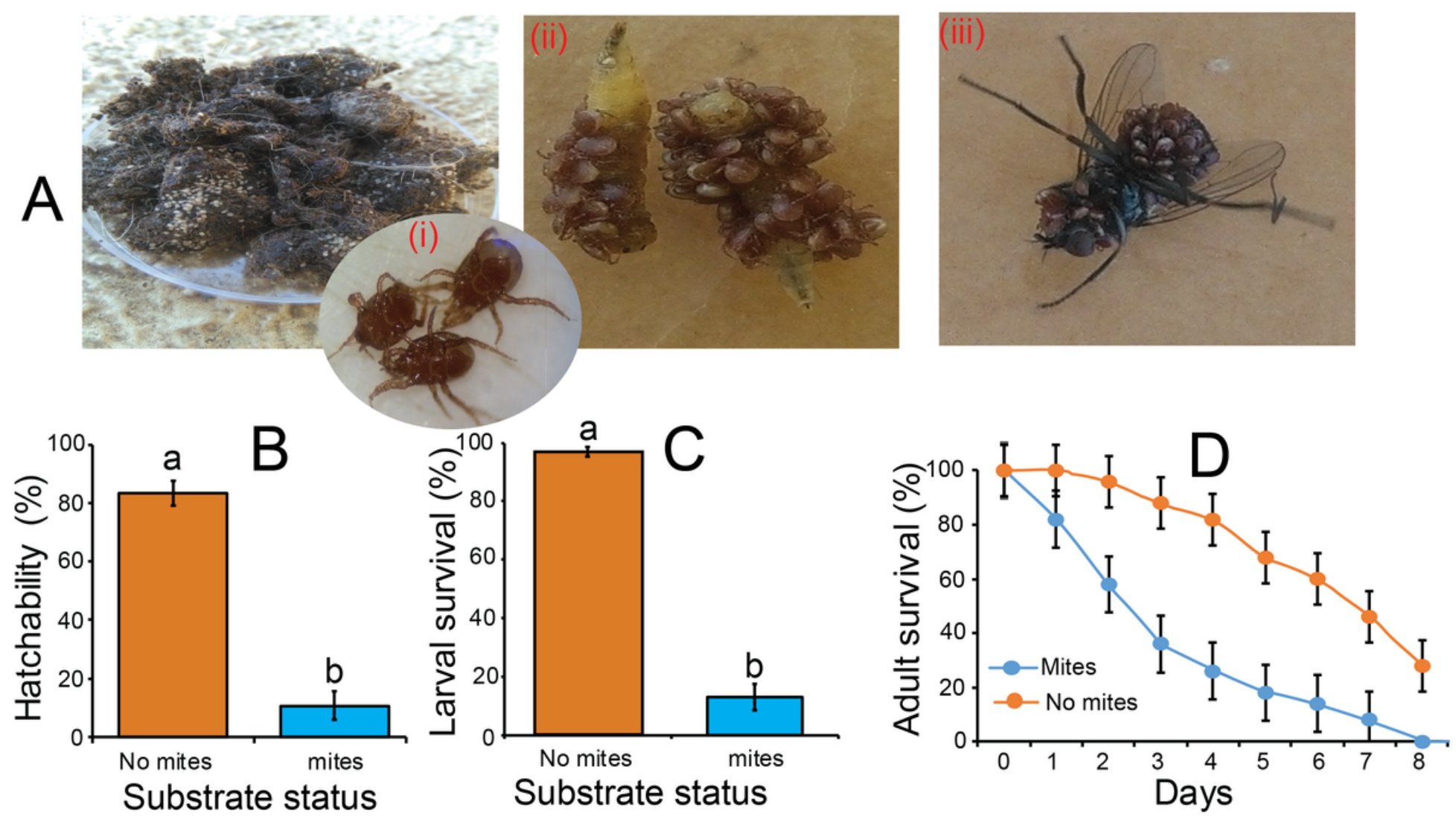

\section{Figure 5}

Effect of the mite M. muscaedomesticae on S. calcitrans survival. (A) Adult M. muscaedomesticae (i) aggregated on dead S. calcitrans larvae as well as (ii) dead and (iii) live adult S. calcitrans. (B-C) Bar charts showing hatchability of S. calcitrans eggs (B) and larval survival (C) on substrates with and without mites (GLM with binomial distribution followed by the analysis of deviance test, $P<0.05, n=10$ ).

(D) Kaplan-Meier curve showing survivorship over time in adult of S. calcitrans with or without mite infestation (Mantel-Cox log-rank $\chi 2$ test, $\mathrm{P}<0.05, \mathrm{n}=5$ ). Error bars indicate the standard error of the mean. Treatments with different lowercase letters are significantly different from each other. 